\title{
Aplicación de Modelos Matemáticos en Curvas de Ruptura de contaminantes del Biogás
}

\author{
Mathematical Models Applied to Biogas \\ contaminants Breakthrough Curves
}

\section{Aplicação de modelos matemáticos em Curvas de ruptura de poluentes Biogás}

\author{
Oscar Vasco-Echeverri; Margarita Ramírez-Carmona; Julián Gómez-Mojica \\ Universidad Pontificia Bolivariana, Facultad de Ingeniería Química, Centro de Estudios y de Investigación en \\ Biotecnología (CIBIOT), Circular 1a, № 70-01 Medellín, Colombia. \\ *oscar.vasco@upb.edu.co
}

Fecha Recepción: 22 de noviembre de 2015 Fecha Aceptación: 8 de enero de 2016

\begin{abstract}
Resumen
En este proyecto se purificó el biogás empleando columnas de adsorción independientes empacadas con carbón activado, limadura de hierro, caolín y carbonato de calcio. Los experimentos se realizaron en columnas de polipropileno de $2 \mathrm{~cm}$ de diámetro y $9 \mathrm{~cm}$ de longitud. Las curvas de ruptura se construyeron con un flujo de biogás promedio diario de 2,2L. Se tomaron muestras durante 28 días como máximo, a las cuales se cuantificaron la composición del biogás mediante cromatografía de gases y un analizador de gases en línea. Las curvas se ajustaron a tres modelos matemáticos. En el caso del Carbón activado se obtuvo un coeficiente de correlación de 0,9987 y 0,9991 para el Sulfuro de Hidrógeno $\left(\mathrm{H}_{2} \mathrm{~S}\right)$ y el Amoniaco $\left(\mathrm{NH}_{3}\right)$, respectivamente. En el caso de la limadura de hierro se obtuvo un coeficiente de correlación de 0,9945 y 0,9975 para el $\mathrm{H}_{2} \mathrm{~S}$ y $\mathrm{NH}_{3}$, respectivamente. Empleando caolín se obtuvo un coeficiente de correlación de 0,9994 y 0,9885 para el $\mathrm{H}_{2} \mathrm{~S}$ y $\mathrm{NH}_{3}$, respectivamente. Con Carbonato de Calcio se obtuvo un coeficiente de correlación de 0,9988 y 0,9985 para el $\mathrm{H}_{2} \mathrm{~S}$ y $\mathrm{NH}_{3}$, respectivamente. El porcentaje de remoción del $\mathrm{H}_{2} \mathrm{~S}$ y del $\mathrm{NH}_{3}$ del carbón activado fue del $72,6 \%$, la limadura de hierro fue del $74,1 \%$, el caolín fue del $32,7 \%$ y del carbonato de calcio del $65,4 \%$.
\end{abstract}

Palabras clave: Modelos matemáticos, curvas de ruptura, purificación de biogás, sulfuro de hidrógeno, amoniaco, adsorción.

\begin{abstract}
In this project biogas was purified using separate packed bed adsorption columns using activated carbon, iron filings, kaolin and calcium carbonate. Experiments were performed in $2 \mathrm{~cm}$ diameter and $9 \mathrm{~cm}$ length polypropylene columns. The breakthrough curves were constructed with an average biogas flow of $2.2 \mathrm{~L}$ per day. Samples were taken for almost 28 days, which biogas composition were quantified using gas chromatography and online gas analyser. The curves were fitted to three mathematical models. Using activated carbon a correlation coefficient of 0.9987 and 0.9991 for hydrogen sulfide $\left(\mathrm{H}_{2} \mathrm{~S}\right)$ and ammonia $\left(\mathrm{NH}_{3}\right)$, respectively, were obtained. In the case of iron filings a correlation coefficient of 0.9945 and 0.9975 for $\mathrm{H}_{2} \mathrm{~S}$ and $\mathrm{NH}_{3}$, respectively, were obtained. Using kaolin a correlation coefficient of 0.9994 and 0.9885 for $\mathrm{H}_{2} \mathrm{~S}$ and $\mathrm{NH}_{3}$, respectively, were obtained. Finally, using calcium carbonate with a correlation coefficient of 0.9988 and 0.9985 for $\mathrm{H}_{2} \mathrm{~S}$ and $\mathrm{NH}_{3}$, respectively, were obtained. Removal percentage of
\end{abstract}

Cita: Vasco-Echeverri O, Ramírez-Carmona M, Gómez-Mojica J. Aplicación de Modelos Matemáticos en Curvas de Ruptura de contaminantes del Biogás. rev.ion. 2016;29(1):81-94. 
$\mathrm{H}_{2} \mathrm{~S}$ and $\mathrm{NH}_{3}$ using activated carbon was $72.6 \%$, iron filings was $74.1 \%$, kaolin was $32.7 \%$ and calcium carbonate was $65.4 \%$.

Keywords: Mathematical models, breakthrough curves, biogas purification, Hydrogen sulfide, ammonia, adsorption.

\section{Resumo}

Nesta pesquisa foi purificado o biogás utilizando colunas de adsorção independentes empacotadas com carvão ativado, limalha de ferro e caulim. Os ensaios foram realizados em colunas de polipropileno de $2 \mathrm{~cm}$ de diâmetro e $9 \mathrm{~cm}$ de comprimento. As curvas de ruptura foram construídas com fluxo médio diário de 2,2L. As amostras foram retiradas durante 28 dias, as quais foram quantificadas a composição de biogás através de cromatografia de gás e um analisador de gás on-line. As curvas foram ajustadas a três modelos matemáticos. Os resultados mostraram para carvão ativado um coeficiente de correlação de 0,9987 e 0,9991 de sulfeto de hidrogênio $\left(\mathrm{H}_{2} \mathrm{~S}\right)$ e amônia $\left(\mathrm{NH}_{3}\right)$, respetivamente. No caso de limalha de ferro um coeficiente de correlação de 0,9945 e 0,9975 de $\mathrm{H}_{2} \mathrm{~S}$ e $\mathrm{NH}_{3}$, respectivamente. Para o caulim um coeficiente de correlação de 0,9994 e 0,9885 de $\mathrm{H}_{2} \mathrm{~S}$ e $\mathrm{NH}_{3}$, respectivamente. Para o carbonato de cálcio um coeficiente de correlação de 0,9988 e 0,9985 de $\mathrm{H}_{2} \mathrm{~S}$ e $\mathrm{NH}_{3}$, respectivamente. A percentagem de remoção de carvão ativado foi de $72,6 \%$, limalha de ferro foi de $74,1 \%$, caulim foi de $32,7 \%$, e carbonato de cálcio foi de $65,4 \%$. Todas as curvas foram ajustadas para o mesmo modelo.

Palabras-chave: Modelos matemáticos, curvas de ruptura, purificação de biogás, sulfeto de hidrogênio, amônia, adsorção.

\section{Introducción}

El biogás es un gas combustible que se genera en medios naturales como pantanos, rellenos sanitarios, estómagos de rumiantes o de manera artificial en dispositivos específicos llamados digestores anaerobios; los microorganismos consumen el componente orgánico degradable obteniéndose biogás y un producto biológicamente estable [1]. Es un combustible de mediano contenido energético $(22-30 \mathrm{MJ} / \mathrm{kg})$ [2], donde la calidad y cantidad de biogás producido dependerá de la composición y la naturaleza del residuo utilizado, de la configuración del reactor y la posibilidad de separar las etapas de la digestión [3]. Aunque la composición del biogás varía de acuerdo a la biomasa utilizada, su composición aproximada está entre 40 y $70 \%$ de metano $\left(\mathrm{CH}_{4}\right)$, 20 y $60 \%$ de dióxido de carbono $\left(\mathrm{CO}_{2}\right), 0$ y $10 \%$ de sulfuro de hidrógeno $\left(\mathrm{H}_{2} \mathrm{~S}\right), 0$ y $1 \%$ de hidrógeno $\left(\mathrm{H}_{2}\right)$ [4], 0 y $5 \%$ de amoniaco $\left(\mathrm{NH}_{3}\right)$ y menos del $1 \%$ de otros gases [5].

Las emisiones de biogás pueden causar daños al medio ambiente por la presencia del $\mathrm{H}_{2} \mathrm{~S}$ gaseoso, que es perjudicial para seres humanos y animales. A bajas concentraciones, este gas tiene un olor desagradable, como al de huevo podrido, y a altas concentraciones puede ser potencialmente mortal [6]. Por su parte el $\mathrm{NH}_{3}$ presente en el biogás puede ser venenoso si se inhala en grandes cantidades (concentraciones por encima de 50-100ppm), e irritación en los ojos, garganta y nariz en niveles de concentración menores [7]. Por otro lado, el biogás puede ser utilizado para generar energía eléctrica, donde estos contaminantes causan corrosión en las partes metálicas del interior de las máquinas, haciendo necesaria la purificación del biogás a niveles inferiores de los 50ppm de $\mathrm{H}_{2} \mathrm{~S}$ [8].

Desde hace algunos lustros se ha venido estudiando la implementación de diversas alternativas para retirar los contaminantes presentes en el biogás [9], dentro de ellas están los procesos de adsorción química, adsorción física, adsorción en superficie sólida, separación por membrana, separación criogénica, conversión química y procesos biológicos [10]. Una de las alternativas más utilizadas es la adsorción en superficie sólida en columnas de lecho empacado, empleando diversos materiales sólidos, el cual cumple la función de filtro para los contaminantes del biogás como el dióxido de carbono, el sulfuro de hidrógeno, el amoniaco, entre otros, garantizando así un biogás con alto contenido en metano [11]. Muchos de los sustratos utilizados en las columnas de adsorción son la limadura de hierro, óxido de calcio, hidróxido de potasio, carbón activado, y otros minerales puesto que estos presentan características fisicoquímicas que favorecen la capacidad de adsorción frente a los contaminantes [12]. 
Las curvas de ruptura son necesarias para el diseño de las columnas de adsorción para la purificación de corrientes líquidas o gaseosas, gracias a la información sobre el comportamiento dinámico de la concentración del analito en el tiempo [13]. Los modelos matemáticos fueron creados para el ajuste de las curvas de ruptura experimentales y la predicción del proceso de adsorción. Estos modelos son usados para el diseño y escalado de los procesos de separación [14]. El criterio más importante en el diseño de una columna de adsorción es la predicción de la curva de ruptura o la forma del frente de onda de adsorción, lo cual determina la longitud del lecho, la vida útil y su tiempo de regeneración [15].

En este trabajo se aplicaron varios modelos matemáticos para describir la saturación de un adsorbente por medio de las curvas de ruptura. Se seleccionaron tres adsorbentes económicos y fáciles de adquirir a nivel nacional como el carbón activado, la limadura de hierro y el caolín. Se realizaron montajes de columnas empacadas con cada adsorbente y se hizo pasar un flujo de biogás producido en un digestor anaerobio. Se midieron las concentraciones de $\mathrm{H}_{2} \mathrm{~S}, \mathrm{NH}_{3}, \mathrm{CH}_{4}$ y $\mathrm{CO}_{2}$ a la entrada y a la salida de la columna para realizar las respectivas gráficas. Finalmente, se analizaron los modelos para ver cuál se ajustaba mejor.

\section{Metodología experimental}

\section{Producción de biogás}

Para la producción de biogás se hizo uso de un digestor anaerobio de 5L (Armfield, W8), con una modificación en la instalación en la parte superior de un agitador mecánico (IKA, RW 16). El digestor cuenta con sistema de control de temperatura, la cual se mantuvo constante a $34^{\circ} \mathrm{C}$ mediante mantas calefactoras de $200 \mathrm{~W}$, y control de la agitación a 150rpm la cual se encendía 20 minutos cada 2 horas. El digestor se alimentaba diariamente a la misma hora con el mismo sustrato para obtener un volumen y unas propiedades lo más estables posibles.

Los sustratos utilizados fueron residuos de comida del restaurante del bloque 11 de Ingenierías de la Universidad Pontificia Bolivariana sede Medellín.
Se trituraron y se conservaron en bolsas de polietileno en un congelador a $-20^{\circ} \mathrm{C}+/-2^{\circ} \mathrm{C}$. Como inóculo inicial se empleó lodo anaerobio proveniente de la PTAR San Fernando.

\section{Selección de los adsorbentes}

El Carbón activado utilizado se adquirió de Cabot (Darco BG) con un tamaño medio de partícula de $1 \mathrm{~mm}$ (malla 16), densidad aireada de $580 \mathrm{~kg} / \mathrm{m}^{3}$ y una superficie específica de $1,100 \mathrm{~m}^{2} / \mathrm{g}$. La limadura de hierro se obtuvo gratuitamente a partir de los residuos en cerrajerías y negocios donde se manejaba la soldadura de hierro, con una densidad promedio de $1890 \mathrm{~kg} / \mathrm{m}^{3}$. El caolín se adquirió localmente en empresas distribuidoras de productos químicos, y proveniente de las minas de Minerales Industriales en La Unión (Antioquia) con una densidad promedio de $755 \mathrm{~kg} / \mathrm{m}^{3}$. El carbonato de calcio se obtuvo de un proveedor de insumos químicos local, con un tamaño de partícula de $4 \mathrm{~mm}$ (malla 4), densidad aireada de $705 \mathrm{~kg} / \mathrm{m}^{3}$ y una superficie específica de $2000 \mathrm{~m}^{2} / \mathrm{g}$.

\section{Construcción y montaje de las columnas de adsorción}

Para la construcción de las columnas de adsorción se realizó con base a lo descrito por Elizondo y Herrera [16], en el cual se establecen materiales que no reaccionen con ningún compuesto presente en el biogás, tal como vidrio, material polimérico o PVC. Las columnas se construyeron en tubos de polipropileno de $2 \mathrm{~cm}$ diámetro interno y una altura de $9 \mathrm{~cm}$. En los extremos se ubicaron racores plásticos de polietileno, inertes a los componentes del biogás, garantizando la hermeticidad. En la parte inferior de las columnas se colocó una malla plástica para retener cada uno de los sustratos para prevenir una posible obstrucción y garantizar el libre flujo del biogás a través de las columnas. Adicionalmente se implementó en cada columna una zona de redistribución, material de menor granulometría dentro del lecho (Figura 1), para garantizar que el biogás tenga contacto con todo el lecho de la columna y así aumentar el área de contacto, y por ende la transferencia de masa incrementa, como lo describen Ramírez et al. [17]. 


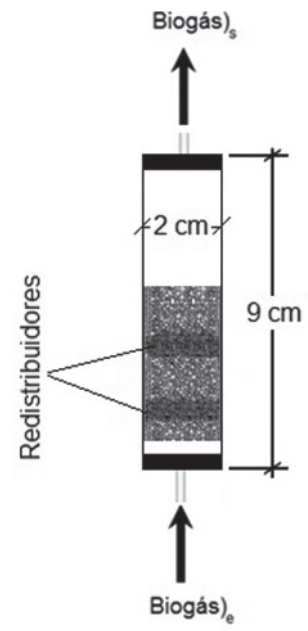

Figura 1. Esquema de la columna empacada construida para el proceso de purificación del biogás.

Las columnas se empacaron en un $40 \%$ de su volumen total con cada sustrato, para garantizar un tiempo de residencia similar debido a la diferencia de densidades. Se utilizaron $3,14 \mathrm{~g}$ de carbón activado, 8,37g de limadura de hierro,
$3,25 \mathrm{~g}$ de caolín y $2,98 \mathrm{~g}$ de carbonato de calcio en las columnas empacadas.

\section{Proceso de purificación del biogás}

En el proceso de purificación del biogás, obtenido del digestor anaerobio (Armfield, W8) de 5L con residuos de restaurante, el cual se conectó la salida de biogás del digestor a la parte inferior de una columna empacada con el adsorbente por medio de tubería flexible de silicona. En el digestor se tomaron directamente las muestras de biogás producido antes de pasar por la columna de adsorción. Luego se conectó la salida de la columna, parte superior, a un colector de biogás provisto para la medición del biogás producido por desplazamiento de un líquido que corresponde a una solución salina ácida preparada con naranja de metilo, $\mathrm{NaCl}, \mathrm{NaHCO}_{3}$ y $\mathrm{H}_{2} \mathrm{SO}_{4}$, para evitar la solubilización de los gases y el cambio de su composición. Desde este reservorio se tomaron las muestras de biogás purificada. El montaje del sistema de purificación del biogás se muestra en la Figura 2.

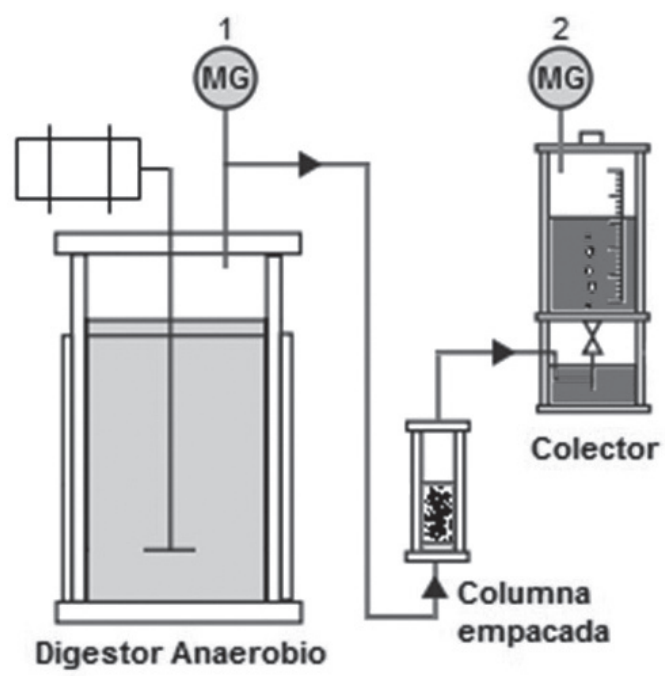

\section{(MG) Muestra de gas}

Figura 2. Esquema del proceso de purificación del biogás.

Las muestras fueron tomadas con una jeringa de polipropileno de $50 \mathrm{~mL}$ del punto MG1, muestra de entrada a la columna empacada, y MG2, salida de la columna empacada, cada 24 horas y llevadas a un cromatógrafo de gases (Agilent 7879A), empleando el método análogo al utilizado por Molinuevo-Salces et al. [18] y a un analizador en línea (Gasdata, GFM400) para la cuantificación de amoniaco $\left(\mathrm{NH}_{3}\right)$, sulfuro de hidrógeno $\left(\mathrm{H}_{2} \mathrm{~S}\right)$, dióxido de carbono $\left(\mathrm{CO}_{2}\right)$ y metano $\left(\mathrm{CH}_{4}\right)$.

\section{Modelamiento de las curvas de ruptura}

Se graficaron las curvas de ruptura para cada sustrato-analito como lo describen Ramírez et al. [17], determinándose la relación de la concentración de salida y entrada de la columna 
cada día. Para la simulación de las curvas de ruptura se emplearon los modelos descritos por Chu [19]. Los modelos 1-3 se observan en las Ecuaciones 1-3, respectivamente.

$$
\begin{array}{r}
\frac{C_{S}}{C_{e}}=\frac{1}{2}\left(1+\operatorname{erf}\left[\frac{t-t_{o}}{\sqrt{2} \cdot \sigma \cdot t_{o}}\right]\right) \\
\frac{C_{S}}{C_{e}}=\frac{1}{2}\left(1+\operatorname{erf}\left[\frac{\left(t-t_{o}\right) \cdot \exp \left(\sigma \cdot\left(\frac{t}{t_{o}}\right)\right)}{\sqrt{2} \cdot \sigma \cdot t_{o}}\right]\right) \\
\frac{C_{S}}{C_{e}}=\frac{1}{2}\left(1+\operatorname{erf}\left[\frac{\left(t-t_{o}\right) \cdot \exp \left(-\sigma \cdot\left(\frac{t}{t_{o}}\right)\right)}{\sqrt{2} \cdot \sigma \cdot t_{o}}\right]\right)
\end{array}
$$

Donde $\operatorname{erf}[x]$ es la función error de $x, t$ es el tiempo, $t_{0}$ es el tiempo donde la concentración de salida es la mitad de la concentración de entrada, $\sigma$ representa la desviación estándar, $C e$ y $C s$ son las concentraciones del analito a la entrada y a la salida, respectivamente. Para observar el modelo que mejor se ajusta a los datos experimentales se realizó un procedimiento presentado por Tejada et al. [20], el cual realiza la suma del cuadrado de los errores y el coeficiente de correlación de los datos experimentales y los modelados.

\section{Resultados y Discusión}

En la selección de los adsorbentes para la purificación del biogás se tuvo presente la capacidad de adsorción del sulfuro de hidrógeno y del amoniaco, dos de los principales contaminantes del biogás, el costo-beneficio y la facilidad para su adquisición nacional o localmente. Con base a sustratos reportados en varios trabajos en la producción de biogás en Colombia, se empleó carbón activado [21] y limadura de hierro [22].

\section{Carbón Activado}

El carbón activado fue seleccionado debido a que es uno de los adsorbentes más empleados por su alta área superficial, volumen de los poros desarrollados, propiedades superficiales únicas y la oportunidad en la modificación química de su superficie [23]. Posee redes interconectadas al azar que proporcionan estructuras de poros internos accesibles altamente desarrollados para la adsorción de contaminantes [24] y productos de interés como la aloína [25]. Muestra afinidad de sustancias polares como $\mathrm{H}_{2} \mathrm{O}, \mathrm{H}_{2} \mathrm{~S}, \mathrm{NH}_{3}, \mathrm{SO}_{2}$, algunos alcoholes, aminas y surfactantes entre otros, debido a la presencia de grupos CarbonoOxígeno en su superficie [26]; en el caso del $\mathrm{H}_{2} \mathrm{~S}$ el carbón activado lo adsorbe y lo descompone en sulfuro elemental y se puede regenerar llevándolo a temperaturas cercanas a los $400^{\circ} \mathrm{C}$ [8]; en el caso del mecanismo de adsorción del $\mathrm{NH}_{3}$ sobre carbón activado, no se ha llegado a una teoría reconocida a nivel mundial, donde actualmente se sigue con el debate [7]. El carbón activado ha sido utilizado tanto a nivel de laboratorio, rural e industrial para el proceso de purificación del biogás [12].

El carbón activado se ha utilizado a nivel mundial para la adsorción de algunos contaminantes del biogás mostrando buenos resultados [27-29]. Pris et al. hablan de su capacidad para remover $\mathrm{H}_{2} \mathrm{~S}$ y compuestos orgánicos volátiles [30], por su parte, Llaneza et al. afirman que al utilizarlo en los procesos de adsorción requiere menos gasto energético, porque se opera a una menor temperatura y presión, donde la reacción se realiza en los poros por lo que el sulfuro de hidrógeno reacciona con el oxígeno produciendo azufre y agua; el azufre queda ocluido en los poros del carbón activado dejando estos sitios inactivos [16]. En los ensayos para la purificación del biogás se inició con la evaluación de carbón activado para la adsorción de dos analitos, el sulfuro de hidrógeno $\left(\mathrm{H}_{2} \mathrm{~S}\right)$ y el amoniaco $\left(\mathrm{NH}_{3}\right)$. Se construyeron las curvas de ruptura acorde a lo descrito por Ramírez et al. [17]. Se graficó la relación de las concentraciones del $\mathrm{H}_{2} \mathrm{~S}$ y el $\mathrm{NH}_{3}$ de entrada y 
salida de la columna de adsorción contra el tiempo del proceso de purificación a un flujo promedio de $91,6 \mathrm{~mL} / \mathrm{h}$ (2,2L/día). Se realizó la comparación de las curvas obtenidas con los tres modelos y con los datos experimentales, Figura 3, como lo desarrollaron Ramírez et al. [13].
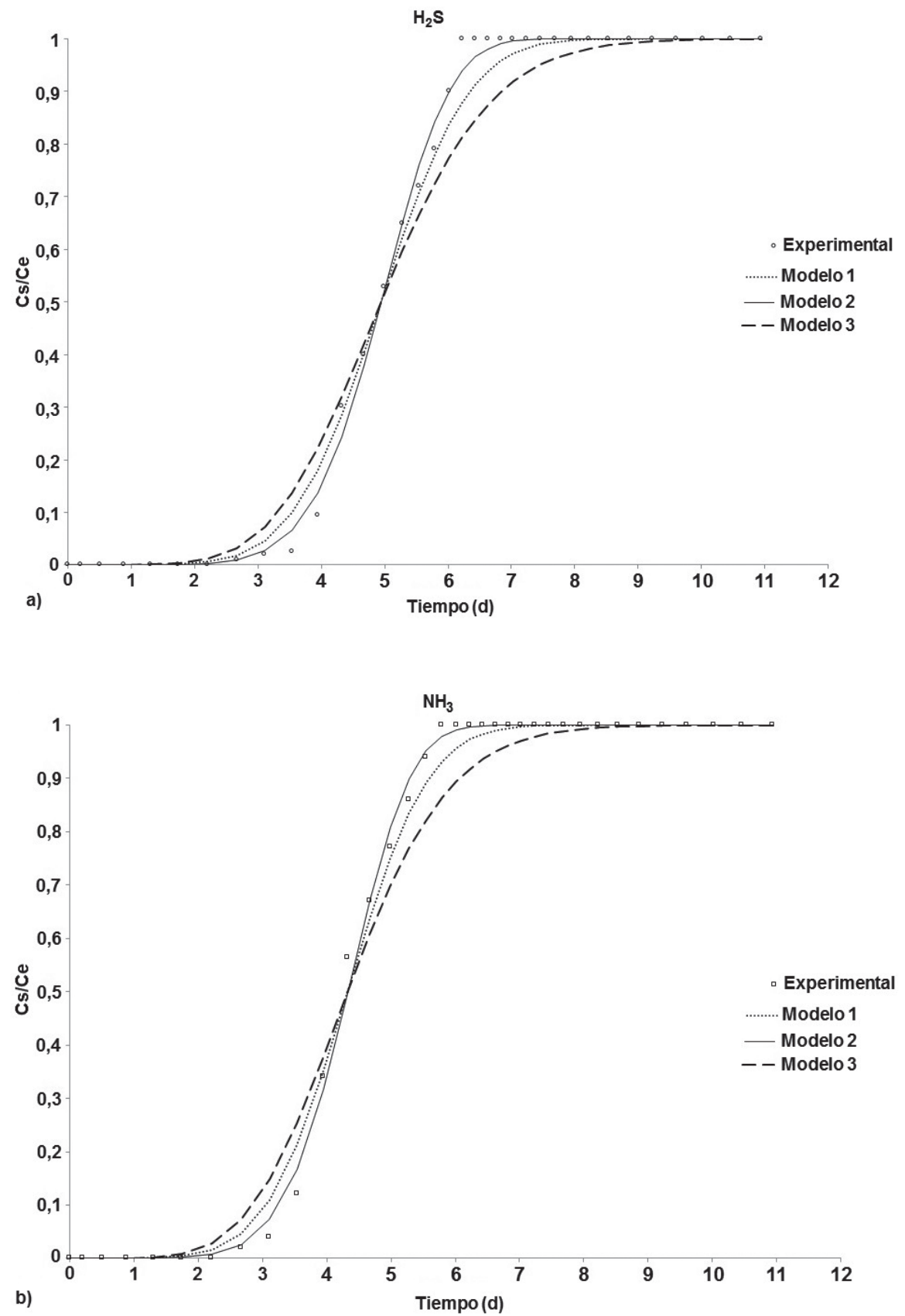

Figura 3. Curvas de ruptura en la adsorción de a) $\mathrm{H}_{2} \mathrm{~S}$ y b) $\mathrm{NH}_{3}$ empleando carbón activado. 
Para la construcción de las curvas de ruptura a partir de los modelos, Ecuaciones 1-3, fue necesario ajustar $t$ y $\sigma$ a partir de la curva de ruptura experimental para cada analito. Los valores de $t_{0} \mathrm{y}$ $\sigma$ empleando carbón activado para el $\mathrm{H}_{2} \mathrm{~S}$ fueron 4,94 días y 0,221 y para el $\mathrm{NH}_{3}$ fueron de 4,32 días y 0,228 , respectivamente. Al comparar los valores de $t_{0}$ y $\sigma$ ajustados para el $\mathrm{H}_{2} \mathrm{~S}$ y $\mathrm{NH}_{3}$, éstos difieren en un $12,5 \%$ y $3,2 \%$, respectivamente, valores cercanos entre si debido al fenómeno de adsorción de los analitos, ya que el carbón activado posee sitios activos, microporos y mesoporos, donde ambos analitos son adsorbidos simultáneamente debido que son polares [31]. Los mesoporos son canales que contienen sitios activos de un tamaño superior a $100 \mathrm{~nm}$ que no son fácilmente bloqueados por los compuestos adsorbidos, una vez los poros se encuentran bloqueados, los sitios activos no están disponibles y ocurre la saturación del soporte donde ocurre el proceso de adsorción [24]. Por tal motivo, el carbón activado se satura con los analitos $\mathrm{H}_{2} \mathrm{~S}$ y $\mathrm{NH}_{3}$ aproximadamente a los 6 días de haber comenzado el proceso de purificación de biogás, utilizando 3,14 gramos de este sustrato a un flujo promedio de biogás de 2,2L/día, con un porcentaje de remoción total del $72,6 \%$, calculadas como indica Ramírez et al. [17], quienes obtuvieron unas eficiencias de remoción del $\mathrm{Cr}^{+3}$ de $53,7 \%$, un $26 \%$ inferior respecto al obtenido en este trabajo, esto debido posiblemente a que la remoción de $\mathrm{NH}_{3}$ está condicionada a la cantidad de $\mathrm{H}_{2} \mathrm{~S}$ o humedad presentes en el biogás como lo demostraron Gonçalves et al. [7], y al adsorberse un analito éste posiblemente mejora la adsorción del otro. Las pendientes de las dos curvas difieren sólo en un $3,2 \%$, y como se había mencionado anteriormente, puede indicar la utilización de los mismos sitios activos dentro del carbón activado por parte de los analitos polares como lo describe Monser y Adhoum [31]. Por otra parte la saturación para los dos analitos fue aproximadamente a los dos días de comenzar el proceso, donde la concentración de entrada en promedio para $\mathrm{H}_{2} \mathrm{~S}$ fue de $165 \mathrm{mg} / \mathrm{L}$ y para el $\mathrm{NH}_{3}$ de $46 \mathrm{mg} / \mathrm{L}$. El incremento promedio del metano fue del 3,3\%, aumento debido a la eliminación de los contaminantes.

En este trabajo se utilizaron los modelos desarrollados por Chu, modelos para la simulación de curvas de ruptura de la adsorción de metales en columnas empacadas [19]. Aunque no se menciona nada acerca de estos modelos para otros tipos de analitos diferentes a los metales, y debido a que la determinación de un modelo predictivo para la adsorción sobre carbón activado es un problema complejo [26], se optó por trabajar con los modelos desarrollados por Chu ya que las isotermas para los sistemas trabajados son favorables. El modelo que mejor se ajustó al proceso de adsorción del $\mathrm{H}_{2} \mathrm{~S}$ y el $\mathrm{NH}_{3}$ al carbón activado fue la Ecuación 2, donde se obtuvo un coeficiente de correlación de 0,9987 y 0,9991 para el $\mathrm{H}_{2} \mathrm{~S}$ y $\mathrm{NH}_{3}$, respectivamente. Aunque la diferencia entre los coeficientes de correlación del modelo 2 con el modelo 1 y modelo 3 fue de 0,1 y $0,6 \%$, respectivamente, lo que indica que los tres modelos describen de forma adecuada el comportamiento de adsorción del $\mathrm{H}_{2} \mathrm{~S}$ y el $\mathrm{NH}_{3}$ por el carbón activado. Los valores de la suma de los errores cuadráticos para la adsorción de $\mathrm{H}_{2} \mathrm{~S}$ empleando carbón activado comparado con los modelos 1 -3 fueron $4,7 \times 10^{-2}, 1,7 \times 10^{-2}$ y $1,5 \times 10^{-1}$, respectivamente, lo que confirma un buen ajuste del modelo 2 al proceso experimental; por su parte, en la adsorción del $\mathrm{NH}_{3}$, la suma de los errores cuadráticos fueron $3,1 \times 10^{-2}, 1,2 \times 10^{-2}$ y $1,1 \times 10^{-1}$ para los modelos 1-3, respectivamente. A partir de estos valores se puede afirmar, siguiendo los lineamientos para la validación de la simulación de las curvas de ruptura [19], que las curvas describen de forma acertada el comportamiento del Carbón activado en la adsorción de los dos analitos, $\mathrm{H}_{2} \mathrm{~S}$ y $\mathrm{NH}_{3}$.

\section{Limadura de Hierro}

Diferentes óxidos y compuestos de hierro han sido utilizados para la remoción de sulfuro de hidrógeno $\left(\mathrm{H}_{2} \mathrm{~S}\right)$ y otros contaminantes del biogás. La reacción del $\mathrm{H}_{2} \mathrm{~S}$ con los óxidos de hierro producen sulfuros insolubles que se depositan en el lecho, con la capacidad de adsorber considerables cantidades de éster [32]. Las torres empacadas con compuestos ferrosos (óxidos e hidróxidos de hierro) para la purificación del biogás ha sido estudiada por varias décadas, y se han utilizado materiales provenientes de talleres donde trabajan con hierro y producen residuos de compuestos férricos [33].

Se construyeron las curvas de adsorción de $\mathrm{H}_{2} \mathrm{~S}$ y $\mathrm{NH}_{3}$ utilizando limadura de hierro $(8,37 \mathrm{~g})$ en una torre empacada con un flujo de biogás $86,4 \mathrm{~mL} / \mathrm{h}$. A partir de los datos experimentales se observó la comparación de los tres modelos matemáticos para el comportamiento de las curvas de ruptura mostrados en la Figura 4. 

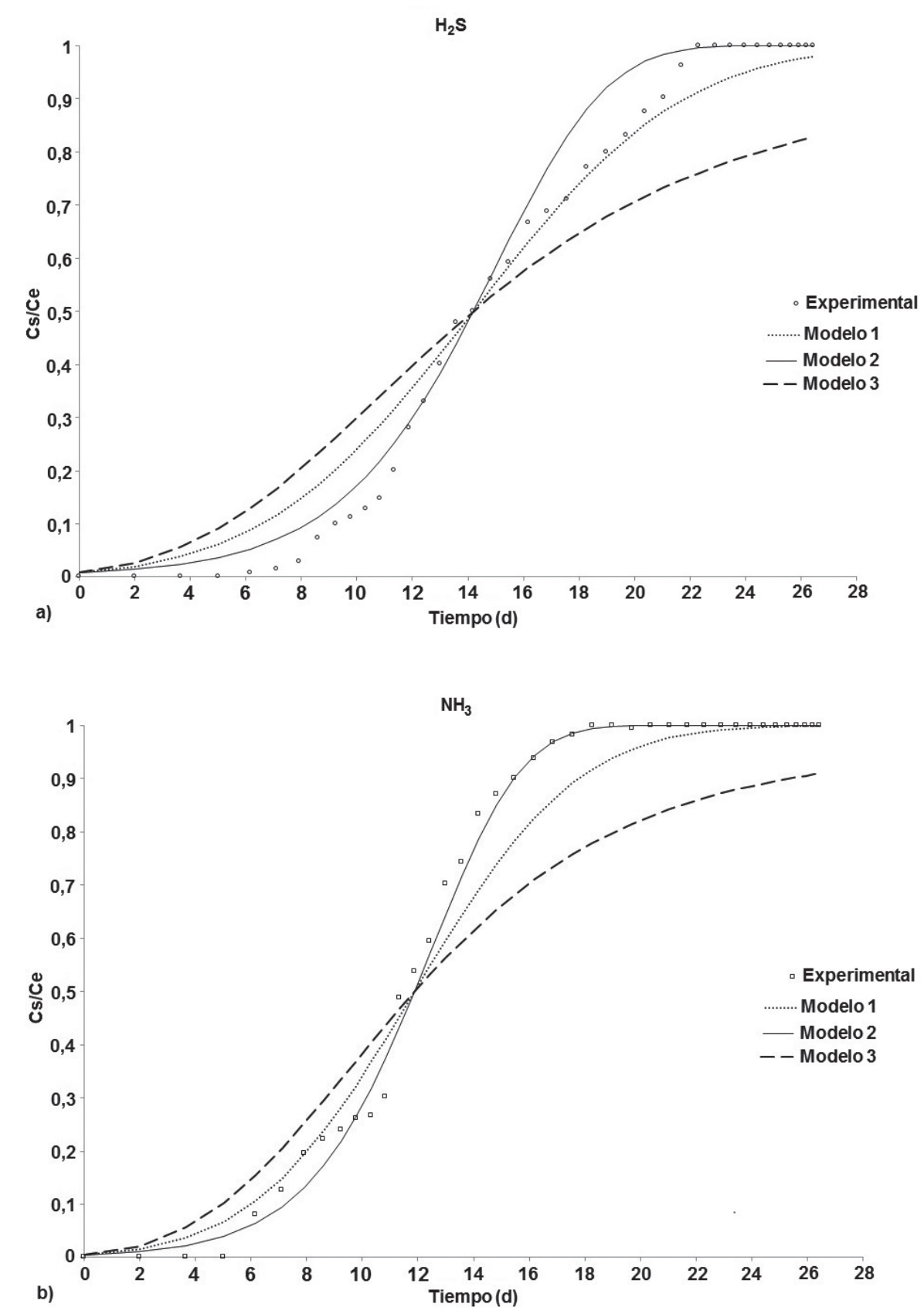

Figura 4. Curvas de ruptura en la absorción de a) $\mathrm{H}_{2} \mathrm{~S}$ y b) $\mathrm{NH}_{3}$ empleando limadura de hierro.

Los valores de $t_{0}$ y $\sigma$ para el $\mathrm{H}_{2} \mathrm{~S}$ de las curvas de ruptura fueron de 14,19 días y 0,419 y para el $\mathrm{NH}_{3}$ fueron de 11,88 días y 0,387 , respectivamente. Los valores de $t_{0}$ y $\sigma$ de los analitos difieren en un $16,2 \%$ y $7,6 \%$, respectivamente. El modelo que mejor se ajustó al proceso de adsorción del $\mathrm{H}_{2} \mathrm{~S}$ y el $\mathrm{NH}_{3}$ empleando limadura de hierro también fue el modelo 2 (Ecuación 2), se obtuvo un coeficiente de correlación de 0,9945 y 0,9975 para el $\mathrm{H}_{2} \mathrm{~S}$ y
$\mathrm{NH}_{3}$, respectivamente. A partir de los valores de los errores absolutos se puede afirmar, siguiendo los lineamientos para la validación de la simulación de las curvas de ruptura [19], que las curvas describen de forma acertada el comportamiento del lecho en la adsorción de los dos analitos.

Los valores de la suma de los errores cuadráticos para la adsorción de $\mathrm{H}_{2} \mathrm{~S}$ empleando limadura de hierro comparados con los modelos 1-3 fueron 
$1,6 \times 10^{-1}, 1,1 \times 10^{-1}$ y $8,9 \times 10^{-1}$, respectivamente, lo que confirma el buen ajuste del modelo 2 al proceso; por su parte, en la adsorción del $\mathrm{NH}_{3}$, la suma de los errores cuadráticos fueron $1,6 \times 10^{-1}, 2,9 \times 10^{-2}$ y $7,6 \times 10^{-1}$ para los modelos $1-3$, respectivamente. A partir de estos valores se puede afirmar que las curvas describen de forma acertada el comportamiento de la limadura de hierro en la adsorción del $\mathrm{H}_{2} \mathrm{~S}$ y el $\mathrm{NH}_{3}$.

\section{Caolín}

El caolín es un mineral extraído directamente de los suelos y es un material compuesto dominativamente por filosilicatos de la familia de la caolinita, pudiendo contener también minerales no filosilicatos tales como el cuarzo además de iones de magnesio, hierro, titanio, potasio y calcio sustituyendo los átomos de aluminio en la estructura [34]. El caolín presenta un tamaño de poro entre 20 y 500 Angstrom, un volumen de poro de $0,06 \mathrm{~cm}^{3} / \mathrm{g}$ y una superficie específica de $4 \mathrm{~m}^{2} / \mathrm{g}$, lo cual resulta beneficioso en aplicaciones como la adsorción [35]. El caolín ha sido investigado para la remoción de $\mathrm{H}_{2} \mathrm{~S}$ y la humedad del gas licuado de petróleo por sus propiedades adsorbentes [36]. Las curvas de adsorción del $\mathrm{H}_{2} \mathrm{~S}$ y el $\mathrm{NH}_{3}$ se construyeron empleando $3,25 \mathrm{~g}$ de caolín en una columna empacada con un flujo de biogás de 2,2L por día (Figura 5).

Los valores de $t_{0}$ y $\sigma$ para el $\mathrm{H}_{2} \mathrm{~S}$ de las curvas de ruptura empleando caolín fueron de 13,27 días y 0,205 y para el $\mathrm{NH}_{3}$ fueron de 8,06 días y 0,618 , respectivamente. Los valores de $t$ y $\sigma$ de los analitos difieren en un $36,2 \%$ y un $201 \%$, respectivamente. En este caso también el modelo que mejor se ajustó fue la Ecuación 2, con un coeficiente de correlación de 0,9994 y 0,9885 para el $\mathrm{H}_{2} \mathrm{~S}$ y $\mathrm{NH}_{3}$, respectivamente. Los valores de la suma de los errores cuadráticos para la adsorción de $\mathrm{H}_{2} \mathrm{~S}$ empleando caolín comparados con los modelos $1-3$ fueron $2,8 \times 10^{-2}, 6,6 \times 10^{-3}$ y $9,2 \times 10^{-2}$ respectivamente, lo que confirma el buen ajuste del modelo 2 al proceso; por su parte, en la adsorción del $\mathrm{NH}_{3}$, la suma de los errores cuadráticos fueron $1,5 \times 10^{-1}, 8,3 \times 10^{-2}$ y 1,6 para los modelos $1-3$, respectivamente. A partir de estos valores se puede afirmar que las curvas describen de forma acertada el comportamiento de la limadura de hierro en la adsorción del $\mathrm{H}_{2} \mathrm{~S}$ y el $\mathrm{NH}_{3}$.

Comparando estos valores con los obtenidos para la limadura de hierro y el carbón activado, se nota que en la modelación del caolín existen diferencias apreciables. En la Figura 5 se observa que el caolín al adsorber simultáneamente el $\mathrm{H}_{2} \mathrm{~S}$ y el $\mathrm{NH}_{3}$, el modelo para éste último no inicia en el origen, mostrando erróneamente que desde el inicio el adsorbente no tiene la capacidad de adsorber el $100 \%$ del $\mathrm{NH}_{3}$ presente en el biogás, lo que no corresponde a la realidad, ya que durante más de dos días la concentración de salida del $\mathrm{NH}_{3}$ en el biogás fue cero. Este fallo en el modelo se debe posiblemente a la ausencia de sitios activos suficientes para adsorber simultáneamente el $\mathrm{H}_{2} \mathrm{~S}$ y el $\mathrm{NH}_{3}$, adsorbiendo durante cerca de ocho días el $\mathrm{H}_{2} \mathrm{~S}$ gracias a la presencia de impurezas como el hierro, el cual posiblemente reacciona mejor con el $\mathrm{H}_{2} \mathrm{~S}$ y no con el $\mathrm{NH}_{3}$. Hosseini menciona que para reducir el hierro presente en el mineral como el caolín hasta una especie soluble, se puede emplear ácidos oxácidos e hidrácidos como agentes oxidantes y oxisales de azufre o cloro como agentes reductores, entre ellos el sulfuro de hidrógeno [37]. Por su parte el $\mathrm{NH}_{3}$ no se ve favorecido en la adsorción empleando caolín, posiblemente a la ausencia de aniones o cationes o las mismas propiedades físicas que si presentan otros materiales como las cenizas del bagazo de caña de azúcar [38]. Sin embargo, se puede decir que las curvas describen de forma acertada el comportamiento del lecho en la adsorción de los dos analitos. El porcentaje de remoción del $\mathrm{H}_{2} \mathrm{~S}$ y del $\mathrm{NH}_{3}$ del caolín fue del $32,7 \%$, siendo éste el más bajo de todos los adsorbentes. 

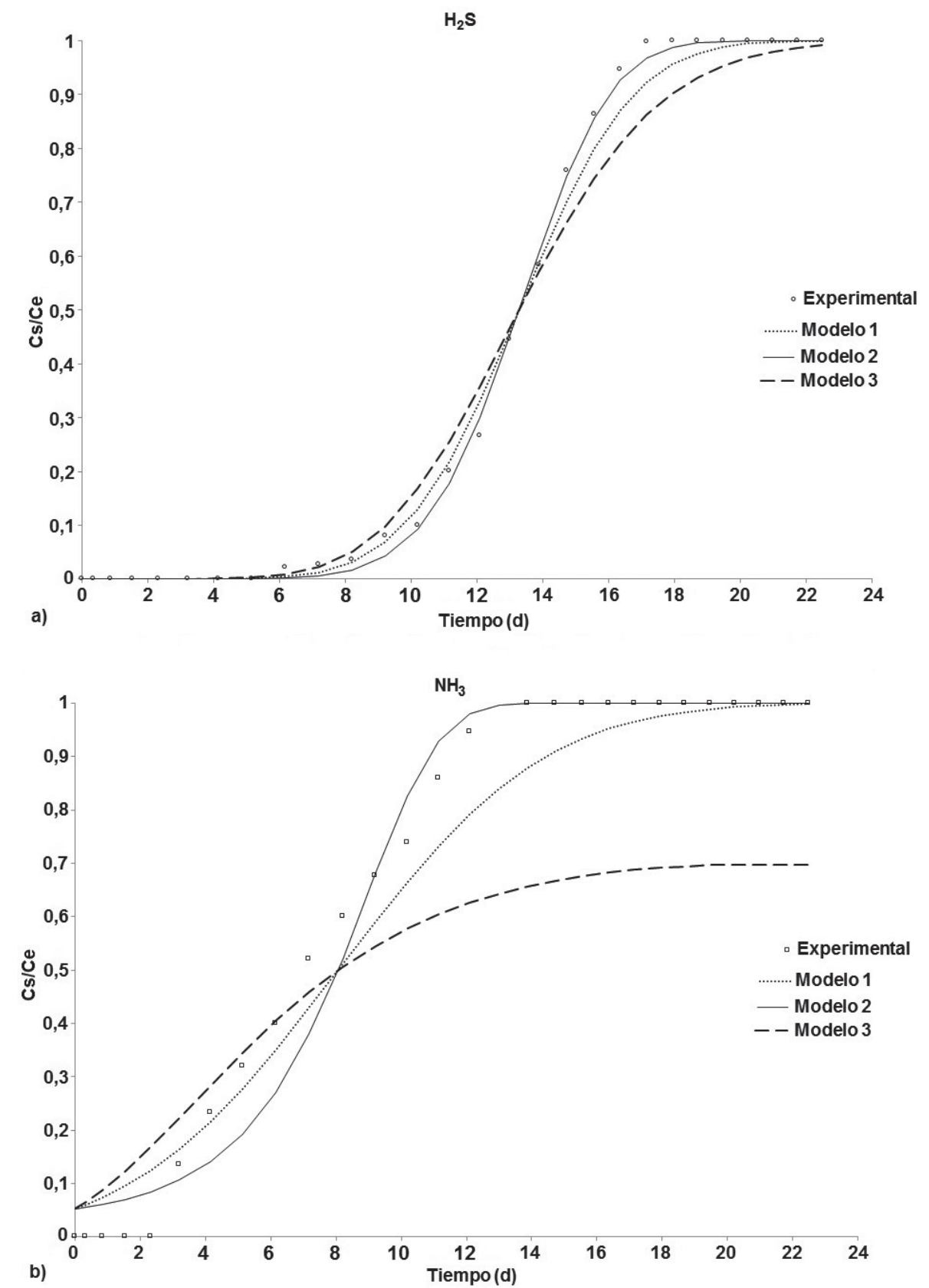

Figura 5. Curvas de ruptura en la absorción de a) $\mathrm{H}_{2} \mathrm{~S}$ y b) $\mathrm{NH}_{3}$ empleando caolín.

\section{Carbonato de calcio}

El Carbonato de calcio es un mineral obtenido por molienda fina de calizas puras con un contenido hasta del $98 \%$ de $\mathrm{CaCO}_{3}$. Cuenta con un área específica entre 200 y $400 \mathrm{~cm}^{2} / \mathrm{g}$, lo que permite una eliminación selectiva de diferentes compuestos ya que es en este sitio donde se dan las interacciones entre los contaminantes y el sustrato [39]. Presenta un volumen de poro de $0,02 \mathrm{~cm}^{3} / \mathrm{g}$ y un tamaño de poro de 105 Angstrom, lo cual resulta beneficioso en aplicaciones como la adsorción [40]. Se han hecho estudios implementando carbonato de calcio como adsorbente de $\mathrm{NH}_{3}, \mathrm{CO}_{2}$ y $\mathrm{H}_{2} \mathrm{~S}$ dada la afinidad presentada [39]. Se construyeron las curvas de adsorción de $\mathrm{H}_{2} \mathrm{~S}$ y $\mathrm{NH}_{3}$ utilizando carbonato de calcio $(3,98 \mathrm{~g})$ en una torre empacada con un flujo de biogás $86,4 \mathrm{~mL} / \mathrm{h}$. A partir de los datos experimentales se observó la comparación de los tres modelos matemáticos para el comportamiento de las curvas de ruptura mostrados en la Figura 6. 


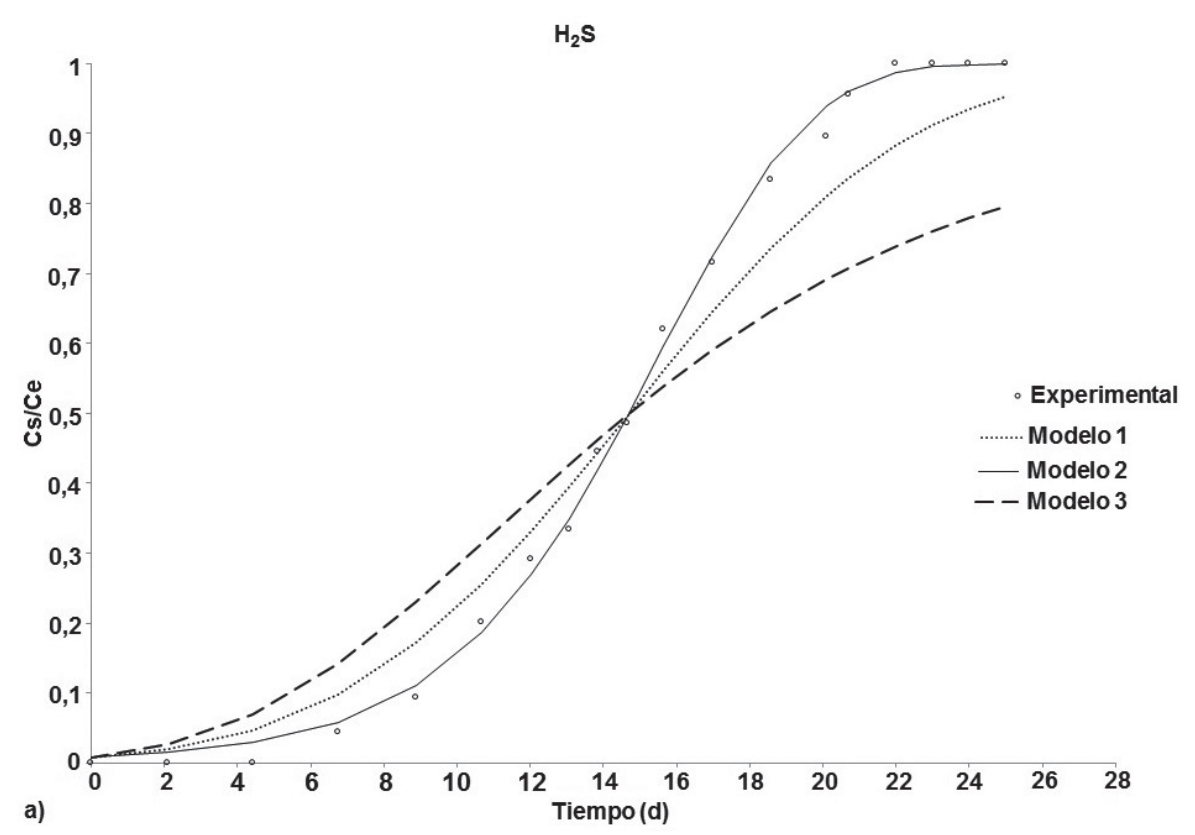

a)

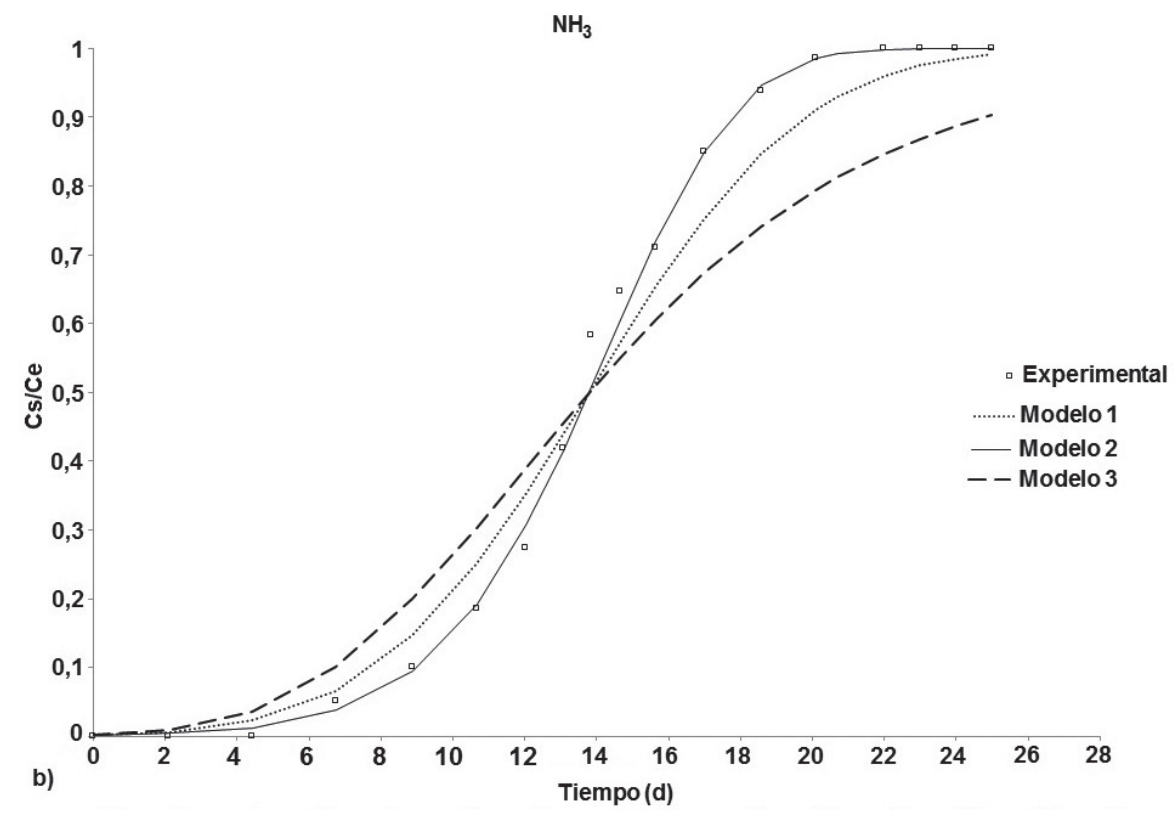

Figura 6. Curvas de ruptura en la absorción de a) $\mathrm{H}_{2} \mathrm{~S}$ y b) $\mathrm{NH}_{3}$ empleando carbonato de calcio.

En la Figura 6 se observa que al emplear carbonato de calcio tiene una tendencia similar que al emplear carbón activado y limadura de hierro, donde el primer analito en saturar el adsorbente es el $\mathrm{NH}_{3} \mathrm{y}$ posteriormente se satura de $\mathrm{H}_{2} \mathrm{~S}$, esto posiblemente debido al tamaño del poro de los adsorbentes o por la afinidad a cada analito [41]. Por otro lado, los valores de $t_{0}$ y $\sigma$ para el $\mathrm{H}_{2} \mathrm{~S}$ de las curvas de ruptura fueron de 14,69 días y 0,417 y para el $\mathrm{NH}_{3}$ fueron de 13,79 días y 0,339 , respectivamente.
Los valores de $t_{o}$ y $\sigma$ de los analitos difieren en un $6,2 \%$ y $18,7 \%$, respectivamente. El modelo que mejor se ajustó al proceso de adsorción del $\mathrm{H}_{2} \mathrm{~S}$ y el $\mathrm{NH}_{3}$ empleando carbonato de calcio fue el modelo 2 (Ecuación 2), y se obtuvo un coeficiente de correlación de 0,9988 y 0,9985 para el $\mathrm{H}_{2} \mathrm{~S}$ y $\mathrm{NH}_{3}$, respectivamente. Los valores de la suma de los errores cuadráticos para la adsorción de $\mathrm{H}_{2} \mathrm{~S}$ empleando carbonato de calcio comparados con los modelos 1-3 fueron $8,9 \times 10^{-2}, 8,1 \times 10^{-3}$ y $4,4 \times 10^{-1}$, 
respectivamente, lo que confirma el buen ajuste del modelo 2 al proceso; por su parte, en la adsorción del $\mathrm{NH}_{3}$, la suma de los errores cuadráticos fueron $5,3 \times 10^{-2}, 1,4 \times 10^{-2}$ y $2,5 \times 10^{-1}$ para los modelos $1-3$, respectivamente. A partir de estos valores se puede afirmar que las curvas describen de forma acertada el comportamiento del carbonato de calcio en la adsorción del $\mathrm{H}_{2} \mathrm{~S}$ y el $\mathrm{NH}_{3}$.

$\mathrm{De}$ los adsorbentes analizados, el mayor porcentaje de remoción de $\mathrm{H}_{2} \mathrm{~S}$ y de $\mathrm{NH}_{3}$ fue al emplear la limadura de hierro con un $74,1 \%$, en segundo lugar fue para el carbón activado con un $72,6 \%$, seguido por el carbonato de calcio con un $65,4 \%$ y el caolín con un $32,7 \%$, mostrando la viabilidad de estos para procesos de purificación de biogás.

\section{Conclusiones}

Se seleccionaron como adsorbentes el carbón activado, la limadura de hierro, el caolín y el carbonato de calcio en el proceso de remoción de $\mathrm{H}_{2} \mathrm{~S}$ y $\mathrm{NH}_{3}$ del biogás y el modelamiento de las curvas de ruptura.

El modelo 2 mostrado en la Ecuación 2, se ajustó correctamente a las curvas de ruptura del carbón activado con un coeficiente de correlación de 0,9987 y 0,9991 para el $\mathrm{H}_{2} \mathrm{~S}$ y el $\mathrm{NH}_{3}$, respectivamente; con la limadura de hierro se obtuvo un coeficiente de correlación de 0,9945 y 0,9975 para el $\mathrm{H}_{2} \mathrm{~S}$ y $\mathrm{NH}_{3}$, respectivamente; empleando caolín se obtuvo un coeficiente de correlación de 0,9994 y 0,9885 para el $\mathrm{H}_{2} \mathrm{~S}$ y $\mathrm{NH}_{3}$, respectivamente; con carbonato de calcio se obtuvo un coeficiente de correlación de 0,9988 y 0,9985 para el $\mathrm{H}_{2} \mathrm{~S}$ y $\mathrm{NH}_{3}$, respectivamente.

De los adsorbentes evaluados, se tiene que el carbón activado y la limadura de hierro presentaron un mejor comportamiento en el proceso de remoción de $\mathrm{H}_{2} \mathrm{~S}$ y $\mathrm{NH}_{3}$ presentes en el biogás, con unos porcentajes de remoción del $74,1 \%$ y del $72,6 \%$, respectivamente.

\section{Referencias bibliográficas}

[1] Rivera González D, Plata Martínez L, Castro Molano L, Guzmán Luna C, Escalante Hernández H. Aprovechamiento del subproducto sólido de la digestión anaerobia del bagazo de fique (furcraea macrophylla) para el acondicionamiento de suelos. rev.ion. 2012;25(1):25-34.

[2] Organización de las Naciones Unidas, Manual de Biogás. Chile; 2011.

[3] Arenas LP, Escalante Hernández H, Combariza MY. Comparative study for methanogenic stage of anaerobic digestion to organic fraction of fruit and vegetable municipal wastes treatment. rev.ion. 2012;25(1):89-96.

[4] Agencia Andaluza de la Energía. Estudio básico del biogás. Sevilla: 2011.

[5] Sanguino Barajas PA, Téllez Anaya NA, Escalante Hernández $\mathrm{H}$, Vasquez Cardozo CA. Aprovechamiento energético de la biomasa residual del sector avícola. revi.ion. 2009;22(1):43-52.

[6] Horikawa MS, Rossi F, Gimenes ML, Costa CMM, Silva MGC. Chemical absorption of H2S for biogas purification. Brazilian J. Chem. Eng. 2004;21(3):415-22.

[7] Gonçalves M, Sánchez-García L, De Oliveira Jardim E, Silvestre-Albero J, RodríguezReinoso F. Ammonia removal using activated carbons: effect of the surface chemistry in dry and moist conditions. Environ. Sci. Technol. 2011;45:10605-10.

[8] Huertas JI, Giraldo N, Izquierdo S. Removal of $\mathrm{H}_{2} \mathrm{~S}$ and $\mathrm{CO}_{2}$ from Biogas by Amine Absorption. In: Mass Transfer in Chemical Engineering Processes. Markoš J, Editor. Croacia: InTech; 2011. p. 134-50.

[9] Tippayawong N, Thanompongchart P. Biogas quality upgrade by simultaneous removal of $\mathrm{CO}_{2}$ and $\mathrm{H}_{2} \mathrm{~S}$ in a packed column reactor. Energy. 2010;35(12):4531-5.

[10] Betzabet Moreno EC, Gropelly E. Revision de las principale tecnologías de prificación de biogás. Cienc. y Tecnol. 2010;10:187-201.

[11] Gupta P, Gupta A. Biogas production from coal via anaerobic fermentation. Fuel. 2014;118:238-42.

[12] Acosta M, Revelo D, Ruiz O, Tax O, Botero R. Capacidad de filtración y retención del sulfuro de hidrógeno $\left(\mathrm{H}_{2} \mathrm{~S}\right)$ del biogás, en dos diferentes sustratos orgánicos (em-compost y lombricompost). Tierra Trop. 2009;5(1):10913.

[13] Ramirez M, Pereira da Silva M, Ferreira SG, Vasco O. Mathematical models applied to the $\mathrm{Cr}(\mathrm{III})$ and $\mathrm{Cr}(\mathrm{VI})$ breakthrough curves. J. Hazard. Mater. 2007;146(1-2):86-90.

[14] Burkert CA, Barbosa GN, Mazutti MA, Maugeri M.Mathematical modeling and experimental breakthrough curves of cephalosporin C adsorption in a fixed-bed column. Process Biochem. 2011;46(6):1270-7. 
[15] Valencia Ríos J, Castellar Ortega G. Predicción de las curvas de ruptura para la remoción de plomo (II) en dis olución acuosa sobre carbón activado en una columna empacada. Fac. Ing. Univ. Antioquia;1:141-58.

[16]Elizondo Quintero AG, Herrera Colindres EN. Evaluación de medios filtrantes para la reducción del sulfuro de hidrógeno en el mejoramiento de la calidad del biogás (Tesis de grado).Tegucigalpa; Honduras: Universidad en Tegucigalpa Zamorano; 2012.

[17] Ramırez Carmona ME, Pereira da Silva MA, Ferreira Leite SG, Vasco Echeverri $\mathrm{OH}$, Ocampo-Lopez C. Packed bed redistribution system for $\mathrm{Cr}(\mathrm{III})$ and $\mathrm{Cr}(\mathrm{VI})$ biosorption by Saccharomyces cerevisia. J. Taiwan Inst. Chem. Eng. 2012;43(3):428-32.

[18]Molinuevo-Salces B, García-González MC, González-Fernández C, Cuetos MJ, Morán A, Gómez X. Anaerobic co-digestion of livestock wastes with vegetable processing wastes: a statistical analysis. Bioresour. Technol. 2010;101(24):9479-85.

[19] Chu KH. Improved fixed bed models for metal biosorption. Chem. Eng. J. 2004;97(2-3):2339.

[20] Tejada Tovar C, Villabona Ortiz A, Ruiz Paternina E. Cinética de adsorción de $\mathrm{Cr}(\mathrm{VI})$ usando biomasas residuales modificadas químicamente en sistemas por lotes y continuo. rev.ion. 2015;28(1):29-41.

[21] Ruiz MB, Gómez P, Giralda R, Carreras Arroyo $\mathrm{N}$, Rodríguez Restrepo M, Ramos Suárez JL. Biogas purification and use as vehicle fuel in rural areas. In: 20th European Biomass Conference and Exhibition. Setting the course for a biobased economy; 2012 Jun 18-22; Milan, Italy; 2012.

[22] Lin W-C, Chen Y-P, Tseng CP. Pilot-scale chemical-biological system for efficient $\mathrm{H}_{2} \mathrm{~S}$ removal from biogas. Bioresour. Technol. 2013;135:283-91.

[23] Xiao Y, Wang S, Wu D, Yuan D. Experimental and simulation study of hydrogen sulfide adsorption on impregnated activated carbon under anaerobic conditions. J. Hazard. Mater. 2008;153(3):1193-200.

[24] Ho N. Modeling Hydrogen Sulfide Adsorption by Activated Carbon made from Anaerobic Digestion By-Product (Master's Theses). Toronto, Canada: University of Toronto; 2012.

[25] Lozano Urbina LA, Muvdi Nova CJ, Mejía Uribe LD. Estabilización del gel de Alor barbadensis
Miller y disminución de su concentración poe adsorción en columna con carbón activado. rev.ion. 2011;24(1):61-7.

[26] Chand Bansal R, Goyal M. Activated Carbon Adsorption. Boca Raton, Estados Unidos: CRC Press, 2005.

[27] Esteves I, Lopes M, Nunes P, Mota J. Adsorption of natural gas and biogas components on activated carbon. Sep. Purif. Technol. 2008;62(2):281-96.

[28] Ho K-L, Lin W-C, Chung Y-C, Chen Y-P, Tseng C-P. Elimination of high concentration hydrogen sulfide and biogas purification by chemical-biological process. Chemosphere. 2013;92(10);1396-401.

[29] Himeno S, Komatsu T, Fujita S. Development of a New Effective Biogas Adsorption Storage Technology. Adsorption. 2005;11(S1):899_ 904.

[30] Hernández SP, Chiappero M, Russo N, Fino D. A novel $\mathrm{ZnO}$-based adsorbent for biogas purification in $\mathrm{H}_{2}$ production systems. Chem. Eng. J. 2011;176-177:272-9.

[31]Monser L, Adhoum N. Modified activated carbon for the removal of copper, zinc, chromium and cyanide from wastewater. Sep. Purif. Technol. 2002;26(2-3):137-46.

[32] Cebula J. Biogas purification by sorption techniques. Archit. Civ. Eng. Environ. 2009;2:95-104.

[33]Zhao Q, Leonhardt E, Macconnell C, Frear C, Chen S. Purification Technologies for Biogas Generated by Anaerobic Digestion. In Compressed Biomethane, CSANR, Ed. 2010.

[34] Mockovčiaková A, Iveta Š, Jiří Š, Ivana K. Characterization of changes of low and high defect kaolinite after bioleaching. Appl. Clay Sci. 2008;39(3-4):202-7.

[35] Li T, Shu Z, Zhou Z, Chen Y, Yu D, Yuan X, Wang Y. Template-free synthesis of kaolinbased mesoporous silica with improved specific surface area by a novel approach. Appl. Clay Sci. 2015;107:182-7.

[36] Ozekmekci M, Salkic G, Fellah MF. Use of zeolites for the removal of $\mathrm{H}_{2} \mathrm{~S}$ : A mini-review. Fuel Process. Technol. 2015;139:49-60.

[37] Hosseini MR, AhmadiA. Biological beneficiation of kaolin: A review on iron removal. Appl. Clay Sci. 2015;107:238-45.

[38] Prieto-García J, Quintana-Puchol R, RodríguezDíaz J, Arteaga-Pérez L, Mollineda-Trujillo A. Estudio termodinámico de la adsorción de amoniaco en ceniza de bagazo de caña de 
azúcar. Rev. Cuba. Química. 2012;24(2):181-4.

[39] Salazar Cuaila JL, Huertas Cardoso J. Evaluación de la eliminación de $\mathrm{CO}_{2}$ y $\mathrm{H}_{2} \mathrm{~S}$ por endulzamiento de biogás usando soluciones acuosas de alcanolaminas. Perú: Universidad Nacional Jorge Basadre Grohmann; 2012.

[40] Castillo Villabona D, Valderrama Solano A. Estudio de Pre-factibilidad Técnica y Economía de la utilización de Biogás en Plantas Diesel para Generación Eléctrica. Colombia: Universidad Industrial de Santander, 2004.

[41] Molina-Sabio M, González JC, RodríguezReinoso F. Adsorption of $\mathrm{NH}_{3}$ and $\mathrm{H}_{2} \mathrm{~S}$ on activated carbon and activated carbonsepiolite pellets. Carbon N. Y. 2004;42(2):44850.

[42]Huynh Q, Thieu VQQ, Dinh TP, Akiyoshi $\mathrm{S}$. Removal of hydrogen sulfide $\left(\mathrm{H}_{2} \mathrm{~S}\right)$ from biogas by adsorption method. In: $8^{\text {th }}$ Biomas Asia Workshop; 2011 nov 29 - dec 1; Hanoi,
Vietnam; 2011.

[43] del Valle Morero B. Purificación de biogás con tecnologías de produción limpias (Tesis de doctorado). Santa Fe, Argentina: Universidad Nacional del Litoral; 2014.

[44] Cherosky P, Li Y. Hydrogen sulfide removal from biogas by bio-based iron sponge. Biosyst. Eng. 2013;114(1):55-59.

[45] Montalvo Martinez SJ, Feernández Santana E. Métodos Económicos y Ecologicamente Viables para Purificar Gases Contaminantes. La Habana, Cuba: Instituto Superior Politécnico José Antonio Echeverría (ISPJAE).

[46]Marín Marín ML. Purificación biológica de biogás. Chile: Universidad de Chile; 2011.

[47]Rakmak N, Wiyaratn W, Chungsiriporn J. Removal of $\mathrm{H}_{2} \mathrm{~S}$ from Biogas by Iron $\left(\mathrm{Fe}^{3+}\right)$ Doped $\mathrm{MgO}$ on Ceramic Honeycomb Catalyst using Double Packed Columns System. Eng. J. 2010;14(1):15-24. 